\title{
Alpha and theta band dynamics related to sentential constraint and word expectancy
}

\author{
Joost Rommers, Danielle S. Dickson, James J. S. Norton, Edward W. Wlotko \& \\ Kara D. Federmeier
}

To cite this article: Joost Rommers, Danielle S. Dickson, James J. S. Norton, Edward W. Wlotko \& Kara D. Federmeier (2017) Alpha and theta band dynamics related to sentential constraint and word expectancy, Language, Cognition and Neuroscience, 32:5, 576-589, DOI: 10.1080/23273798.2016.1183799

To link to this article: http://dx.doi.org/10.1080/23273798.2016.1183799

$$
\text { 曲 Published online: } 19 \text { May } 2016 .
$$

Submit your article to this journal




\title{
Alpha and theta band dynamics related to sentential constraint and word expectancy
}

\author{
Joost Rommers ${ }^{\mathrm{a}, \mathrm{b}}$, Danielle S. Dickson ${ }^{\mathrm{a}}$, James J. S. Norton ${ }^{\mathrm{b}, \mathrm{c}}$, Edward W. Wlotko ${ }^{\mathrm{d}}$ and Kara D. Federmeier ${ }^{\mathrm{a}, \mathrm{b}, \mathrm{c}}$ \\ ${ }^{a}$ Department of Psychology, University of Illinois, Urbana-Champaign, IL, USA; ${ }^{b}$ Beckman Institute for Advanced Science and Technology, \\ University of Illinois, Urbana-Champaign, IL, USA; 'Program in Neuroscience, University of Illinois, Urbana-Champaign, IL, USA; ${ }^{d}$ Sackler School of \\ Graduate Biomedical Sciences, Tufts University, Boston, MA, USA
}

\begin{abstract}
Despite strong evidence for prediction during language comprehension, the underlying mechanisms, and the extent to which they are specific to language, remain unclear. Re-analysing an event-related potentials study, we examined responses in the time-frequency domain to expected and unexpected (but plausible) words in strongly and weakly constraining sentences, and found results similar to those reported in nonverbal domains. Relative to expected words, unexpected words elicited an increase in the theta band $(4-7 \mathrm{~Hz})$ in strongly constraining contexts, suggesting the involvement of control processes to deal with the consequences of having a prediction disconfirmed. Prior to critical word onset, strongly constraining sentences exhibited a decrease in the alpha band $(8-12 \mathrm{~Hz})$ relative to weakly constraining sentences, suggesting that comprehenders can take advantage of predictive sentence contexts to prepare for the input. The results suggest that the brain recruits domain-general preparation and control mechanisms when making and assessing predictions during sentence comprehension.
\end{abstract}

ARTICLE HISTORY

Received 20 December 2015

Accepted 15 April 2016

\section{KEYWORDS}

Prediction; language; sentence comprehension; pre-stimulus alpha; frontal theta

\section{Introduction}

Language arrives in the form of a rapid input stream that needs to be decoded at high speed. Comprehenders seem to experience little difficulty in accessing meanings from memory and ultimately reconstructing the intended message, despite reading at speeds of 250 words per minute (e.g. Rayner, 1998) or listening to more than 200 syllables per minute (e.g. Robb, Maclagan, $\&$ Chen, 2004).The rapidity and seeming ease with which people comprehend language may be afforded in part by the fact that language is, to some extent, predictable. Indeed, current views on language comprehension emphasise processes that help comprehenders keep up with the rapidity of the input by capitalising on its predictability (Altmann \& Mirković, 2009; Christiansen \& Chater, 2015; Dell \& Chang, 2014; Federmeier, 2007; Kamide, 2008; Kleinschmidt \& Jaeger, 2015; Kutas, DeLong, \& Smith, 2011; Levy, 2008; Pickering \& Garrod, 2013). There is now strong evidence that listeners and readers can predict aspects of upcoming information, including syntactic structure (Arai \& Keller, 2013; Carminati, Van Gompel, Scheepers, \& Arai, 2008; Staub \& Clifton, 2006), aspects of word meaning (Altmann \& Kamide, 1999; Federmeier \& Kutas, 1999; Rommers, Meyer, Praamstra, \& Huettig, 2013), and even specific words (van Berkum, Brown, Zwitserlood, Kooijman, \& Hagoort, 2005; DeLong, Urbach, \& Kutas, 2005; Laszlo \& Federmeier, 2009; Wicha, Moreno, \& Kutas, 2004). Despite this progress, the cognitive and neurophysiological mechanisms underlying predictive language processing remain unclear.

In particular, because language processing is only one of many cognitive functions that can benefit from the formation of expectations about upcoming input, it is not clear to what extent prediction-related processes in language may arise from domain-general mechanisms. It has been suggested that prediction in language could be based on event representations common to the linguistic and non-linguistic domains (Altmann \& Mirković, 2009). Furthermore, recent attempts to characterise language processing rely on forward models inspired by action and perception research (Pickering \& Garrod, 2013) or on domain-general neurophysiological principles (Friederici \& Singer, 2015; see also Lewis, Wang, \& Bastiaansen, 2015). In fact, some have argued that minimising prediction error (the difference between what is predicted and what is actually presented) is a fundamental principle of brain function that explains action, perception, and learning (Clark, 2013; Friston, 2010). 
Any prediction-related mechanisms that are not specific to language would presumably manifest in neural signatures that look similar across verbal and nonverbal tasks. Such mechanisms might be visible in modulations of rhythmic, oscillatory brain activity, which can be visualised in spectrograms of the electroencephalogram (EEG). EEG oscillations are believed to reflect the dynamic coupling and uncoupling of neuronal networks (Singer, 1993). Their rhythmic fluctuations may produce temporal windows for communication between brain areas (Fries, 2005), the timing of which can be optimised in anticipation of upcoming information - including, for example, speech sounds (e.g. Arnal \& Giraud, 2012).

Most electrophysiological studies of sentence processing have relied on event-related potentials (ERPs), which are formed by extracting epochs time-locked to events from the continuous data, aligning them and averaging them point-by-point. This successful approach has yielded many core insights into cognition and brain functioning (for an overview, see Luck \& Kappenman, 2011). However, not all of the signal is captured by timedomain averaging, because oscillations that are not phase-locked largely cancel one another in ERPs. Timefrequency analyses help quantify non-phase-locked activity by decomposing the epochs into multiple frequencies and extracting the power at each frequency over time. When such analyses are applied to individual trials, and the spectrograms are averaged together, not only phase-locked, but also non-phase-locked, activity can be assessed (e.g. Tallon-Baudry \& Bertrand, 1999). Different frequency bands, including theta $(4-7 \mathrm{~Hz})$, alpha $(8-12 \mathrm{~Hz})$, beta $(13-30 \mathrm{~Hz})$, and gamma $(>30 \mathrm{~Hz})$, have been associated with different responses to cognitive manipulations. The present study investigated spectro-temporal EEG modulations related to two aspects of sentence comprehension, using an existing dataset for which ERPs have already been assessed. First, we examined differences related to the apprehension of expected and unexpected words, to determine the impact of predictability on processing once words have been encountered. Second, we asked how readers use sentence contexts of differing predictive strengths to prepare for upcoming words before they are presented.

\section{Spectro-temporal signatures of processing unexpected words}

The processing of unexpected stimuli has most often been investigated in nonverbal tasks, where it is known to elicit fronto-centrally distributed power increases in the theta band (e.g. Luu \& Tucker, 2001). This theta increase appears to be a common response across manipulations associated with novelty, conflict, and error, despite these manipulations eliciting different responses in the ERP (for review, see Cavanagh \& Frank, 2014). Frontal theta increases in these various tasks are thought to reflect a shared need for enhanced cognitive control, which serves as a signal to adapt behaviour during learning, possibly based on prediction error (Cavanagh, Frank, Klein, \& Allen, 2010; van de Vijver, Ridderinkhof, \& Cohen, 2011).

In verbal tasks, some studies of sentence processing have examined the spectral response to unexpected words in the form of different types of anomalies. Relative to congruent words, semantically anomalous words (e.g. "gender" in "The industrious employee copies the gender for the impatient boss") have also been found to elicit theta band power increases (Bastiaansen \& Hagoort, 2015; Wang, Zhu, \& Bastiaansen, 2012). Sometimes, these theta increases also have a frontal distribution, although the language literature has tended to interpret them as reflecting lexical-semantic retrieval rather than error processing (e.g. Bastiaansen, van der Linden, terKeurs, Dijkstra, \& Hagoort, 2005; Hald, Bastiaansen, \& Hagoort, 2006). Some studies additionally observed beta band decreases in response to both semantic anomalies (Luo, Zhang, Feng, \& Zhou, 2010; Wang, Jensen, et al., 2012) and syntactic anomalies (e.g. "throw" in "The spoiled little kid throw the toy on the ground"; Bastiaansen, Magyari, \& Hagoort, 2010; Davidson \& Indefrey, 2007). Moreover, gamma band power increases present over the course of a sentence are disrupted when an anomalous word is encountered (Hald et al., 2006; Peña \& Melloni, 2012; Rommers, Dijkstra, \& Bastiaansen, 2013; Wang, Zhu, et al., 2012). Thus, several frequency bands seem to be relevant for dealing with unexpected words.

The extant time-frequency data on written sentence comprehension come from experiments that were not explicitly designed to investigate predictive language processing. For instance, a semantic anomaly is a very specific type of unexpected word, which is generally surprising in its context regardless of whether or not a prediction has been made (and any prediction error-related signals it might generate would presumably not form a useful basis for adaptive learning). Another contrast, that between expected words and plausible alternatives in medium to strongly constraining sentences (Wang, Zhu, et al., 2012), comes closer to addressing our specific question, but some of the plausible alternatives used in this study seem to be semantically related to the expected words, and as such may not have disconfirmed broad semantic expectations. Here, therefore, we examined the spectro-temporal response within a data set that contains sentences that end with either expected 
Table 1. Example item.

\begin{tabular}{llll}
\hline Constraint & \multicolumn{1}{c}{ Sentence frame } & Expected & Unexpected \\
\hline Strong & The children went outside to & play & look \\
Weak & Joyce was too frightened to & move & look \\
\hline
\end{tabular}

words or with words that, while unexpected, are plausible in their sentence contexts, and unrelated to the expected words - which, to our knowledge, have thus far only been analysed using ERPs.

\section{The present study}

We report a re-analysis of an existing ERP study by Federmeier, Wlotko, De Ochoa-Dewald, and Kutas (2007) that distinguished between effects of constraint and expectancy (for functional magnetic resonance imaging results using related information-theoretic measures, see Willems, Frank, Nijhof, Hagoort, \& Van den Bosch, 2015). Importantly, constraint is defined at the level of the sentence frame, based on the cloze probability of the best completion for that sentence. Cloze probability of a word in a sentence fragment is usually defined as the proportion of an independent group of participants who complete the sentence fragment with that word in an off-line task. Strong constraint sentences are those that have the possibility of leading to a high cloze probability completion, and, thus, are presumed to be sentences that afford a strong, consistent prediction. In contrast, weak constraint sentences do not strongly bias participants to produce a consistent completion. Expectancy, then, is defined at the word level, and can be manipulated within both strong and weak constraint sentences, as both can end in (somewhat) expected or unexpected words, as shown in Table 1. This design thus allows us to examine how expected and unexpected words are processed in contexts in which a strong prediction could versus could not be made.

The ERPs from this study are shown in Figure 1. There were two primary effects. First, expectancy, as indexed by cloze probability, modulated the amplitude of the N400, a negativity peaking around $400 \mathrm{~ms}$ after word onset, which indexes semantic processing (Kutas \& Hillyard, 1980). Replicating numerous past studies, the amplitude of the N400 was graded with cloze probability, with the smallest N400s to high cloze probability expected endings of strong constraint sentences, an intermediate response to the low-to-moderate cloze probability expected endings of weak constraint sentences, and the largest $\mathrm{N} 400$ to the unexpected words. The N400 to unexpected words was not affected by sentential constraint. In contrast, a late, frontally-distributed
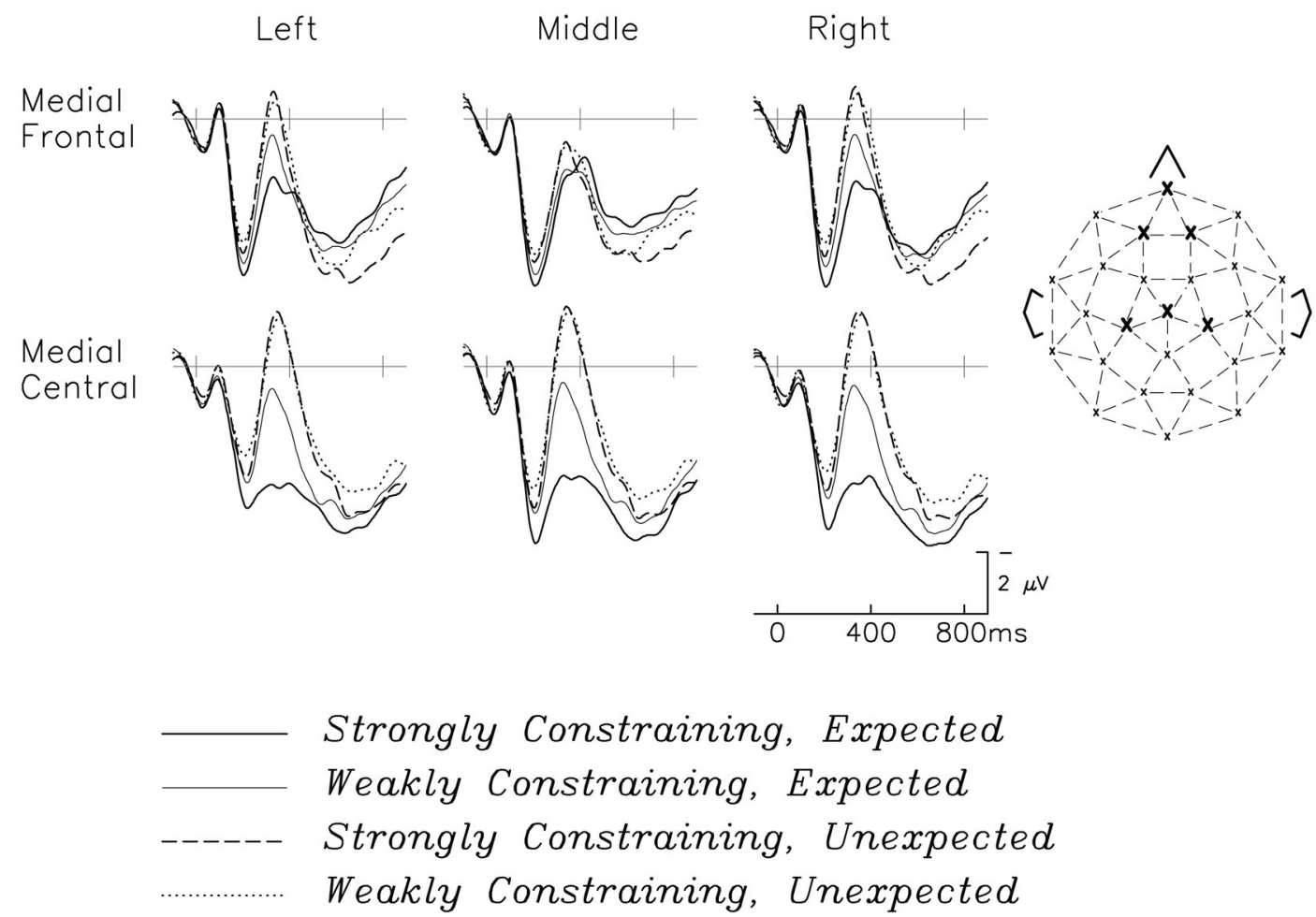

Figure 1. Grand average ERPs at six channels, three over the central part of the head (showing the N400 effect pattern) and three over the front of the head (showing the frontal positivity to unexpected items in strongly constraining contexts). The head diagram on the right shows the positions (with X's) of the electrodes. Reprinted from Federmeier et al. (2007), with permission from Elsevier. 
positivity was selectively enhanced in response to unexpected words in strongly constraining contexts. Because these contexts potentially allow comprehenders to form strong predictions for upcoming words, the late positivity likely reflects some aspect of dealing with disconfirmed predictions (see also DeLong, Quante, \& Kutas, 2014; van Petten \& Luka, 2012).

In analysing the spectral dynamics in these data, we had two main areas of focus. First, we set out to characterise the brain's response in the time-frequency domain to plausible words that disconfirm ongoing expectations, in the hope that this would allow us to connect the findings to the broader literature looking at responses to error and conflict. In doing this, we also wanted to examine the extent to which any elicited power modulations reflect effects distinct from those known to be elicited in the ERP; to that end, we also analysed power changes in the averaged ERP, and compared the results qualitatively (see Methods).

Second, we sought evidence for the use of predictive processing mechanisms prior to the onset of the critical sentence-final words - something that has not been examined in the ERP domain for this data set, and, more generally, which may be unlikely to manifest as activity that is time-locked and phase-locked to word onsets. Thus, we looked for effects of constraint prior to critical word onset, a point in time at which constraint could come into play regardless of expectancy. Recently it has been observed that, relative to weakly constraining sentences, strongly constraining sentence frames can elicit alpha and beta band decreases prior to target pictures, at least when the pictures have to be named (Piai, Roelofs, \& Maris, 2014). A similar effect was visible in the magnetoencephalogram (MEG), and part of this effect remained when participants judged the predictability of the pictures without naming them (Piai, Roelofs, Rommers, \& Maris, 2015). Against this background, we examined whether any constraint effect could be observed prior to the onset of the critical words when the task was simply to read for comprehension. Finally, if such an effect was observed, we planned to use any variability between participants to begin to explore whether there is a relationship between pre-stimulus constraint effects and the subsequent processing of expected and unexpected words.

\section{Methods}

The participants, materials, procedures, and EEG recording parameters described below were previously reported more extensively in Federmeier et al. (2007).

\section{Participants}

The participants were 32 right-handed native speakers of English (16 women and 16 men), with an average age of 20 years (range 18-28 years). Seven participants reported having left-handed or ambidextrous family members. None reported having a history of neurological or psychiatric disorders.

\section{Materials}

The materials consisted of 141 weakly constraining and 141 strongly constraining sentence frames, ending in a critical word. Half of each type of sentence frame ended with an expected word, and half ended with an unexpected word. Table 1 presents an example. The expected words were the most frequent response in an off-line norming in which (different) participants completed sentence frames with the word they would generally expect to be the ending of the sentence fragment. They were also asked to provide two additional plausible endings. Cloze probability of a word in a sentence fragment was defined as the proportion of participants who completed the sentence fragment with that word. Expected words had an average cloze probability of $85.3 \%$ in strongly constraining sentences (all cloze values over 67\%; mean use as next best completion $4.9 \%$ ) and of $26.9 \%$ in weakly constraining sentences (all cloze values under 42\%; mean use as next best completion 9.3\%); these expected words were matched across constraint for word length and frequency by selecting a subset of sentences from the larger set for which cloze probabilities had been collected. Unexpected words were plausible but with cloze probabilities near zero, and were selected to be semantically unrelated to (and not associated with) the most expected word. The unexpected completions of both strongly and weakly constraining sentence frames comprised the same set of words; these had an average cloze probability of $0.6 \%$ in strongly constraining sentences (mean use as next best completion $2.6 \%$ ) and of $1.5 \%$ in weakly constraining sentences (mean use as next best completion 1.6\%). As described in more detail in Federmeier et al. (2007), sentences were matched for length across constraint. The sentences were divided into two lists such that each participant saw each sentence frame only once, and matched unexpected endings did not appear on the same list.

\section{Procedure}

Participants sat at a distance of $100 \mathrm{~cm}$ from a computer screen. On each trial, a warning sign (several pluses) was 
presented for $500 \mathrm{~ms}$, followed by a blank screen for a random duration between 500 and $1200 \mathrm{~ms}$. Then, a sentence was presented word by word in the centre of the screen $(200 \mathrm{~ms}$ per word, followed by $300 \mathrm{~ms}$ of blank screen), followed by a 3000 ms pause. Participants read the sentences for comprehension and were told that they would be asked to answer questions about what they had read at the end of the session. The experiment began with a practice block, after which four experimental blocks were presented, with a short break after each block. After the recording session, participants completed a paper-and-pencil word recognition test, in which they were asked to circle the words that they had read as a final word of a sentence during the experiment. Memory performance showed that participants successfully distinguished between new words and words that they had previously read (see Federmeier et al., 2007).

\section{EEG recording and analysis}

The EEG was recorded from 26 evenly spaced tin electrodes mounted in a cap, referenced online to the left mastoid. Additional electrodes were placed on the right mastoid, the outer canthus of each eye to monitor eye movements, and the infraorbital ridge of the left eye to monitor blinks. Electrode impedances were kept below $5 \mathrm{k} \Omega$. The signal was amplified, filtered with a bandpass from 0.01 to $100 \mathrm{~Hz}$ along with a $60 \mathrm{~Hz}$ notch filter, and digitised at a sampling rate of $250 \mathrm{~Hz}$.

The data were re-referenced to the average of the left and right mastoids. Deviating from Federmeier et al. (2007), the continuous EEG was then segmented into epochs encompassing the signal from $-1700 \mathrm{~ms}$ to $1200 \mathrm{~ms}$ relative to the onset of the sentence endings (i.e. including $1000 \mathrm{~ms}$ of the final word as well as the three immediately preceding words (500 ms each), and $200 \mathrm{~ms}$ of data padding on each side to allow for the time-frequency analysis). Epochs with eye movements, blinks, or other artefacts were removed $(10.6 \%$ of the data). The remaining number of trials per participant per condition was: Strong constraint expected (mean \pm SD) $64 \pm 5$; Strong constraint unexpected $64 \pm 7$; Weak constraint expected $65 \pm 6$; Weak constraint unexpected $65 \pm 6$.

Time-frequency representations of power were computed using the Matlab toolbox Fieldtrip (Oostenveld, Fries, Maris, \& Schoffelen, 2011). The analysis used a moving window short-time Fast Fourier Transform (FFT) approach. The window was $400 \mathrm{~ms}$ long and moved along the time axis in steps of $10 \mathrm{~ms}$ and along the frequency axis in steps of approximately $1 \mathrm{~Hz}$, from 2 to $30 \mathrm{~Hz}$ (higher frequencies were not examined, because the data had been notch-filtered during recording). Each instance of a window was multiplied with a Hanning taper and Fourier transformed. The resulting spectrograms were averaged across trials for each condition and each participant.

Separate analyses were conducted of the signal around the onset of the critical word ( -500 to 1000 $\mathrm{ms})$ and the signal preceding the critical word $(-1500$ to $0 \mathrm{~ms}$ ). For contrasts between the conditions, no baseline correction was performed, but the spectrograms from each condition were divided (element by element) by the average power spectrogram across all four conditions, yielding relative power changes. This prevents pre-stimulus baseline activity from influencing power estimates later in the epoch (although the results did not depend on this analysis choice), and allows for investigation of pre-stimulus activity (e.g. Piai et al., 2014). For visual inspection of power changes in individual conditions during the critical word, power was expressed as a proportion change relative to a baseline from $-500 \mathrm{~ms}$ to $-150 \mathrm{~ms}$ (see Wang, Zhu, et al., 2012 , for previous use of the same settings).

Given the lack of a priori knowledge about the time points, channels, and frequency bands in which effects were expected to occur, statistically significant differences between conditions were identified using nonparametric cluster-based permutation tests (Maris \& Oostenveld, 2007). Briefly, these tests worked as follows with our settings. First, a dependent $t$-test quantified the difference between the conditions at each (time, frequency, channel)-pair, and data points that did not meet a significance level of .05 were zeroed. Then, clusters of data points with a statistically significant difference were formed across neighbouring time, channel, and frequency points, for which the cluster-level $t$ value was the sum of all $t$ values within the cluster, and the cluster with the maximum sum was selected. Next, a null distribution was created by randomly swapping around the condition labels within participants 1000 times and computing the cluster-level $t$ values for each randomisation. Finally, the observed $t$ value was compared with the null distribution. The effect was considered significant when the observed statistic fell within one of the 2.5th percentiles of the null distribution. Because this test was designed to compare two conditions at a time, we separately examined the difference between unexpected and expected words within the strongly constraining contexts and within the weakly constraining contexts. For the analysis of the signal during the critical word, all time and frequency points from critical word onset to $1000 \mathrm{~ms}$ after word onset were submitted to the cluster-based permutation tests, without preselection of time windows, frequency bands, or channels. 
For the analysis of the signal preceding the critical word, we examined the difference between strongly constraining and weakly constraining sentence contexts (averaging across all relevant trials, regardless of expectancy), applying the same statistical test to the signal from $1500 \mathrm{~ms}$ prior to critical word onset up to critical word onset. Finally, we investigated correlations between the various observed effects across subjects, as well as to what extent the power changes were related to ERP effects; these analyses are described with the corresponding results.

\section{Results}

\section{Time-frequency analyses of power}

Overall, sentence endings elicited an early broad band power increase, followed by a theta band increase that partly coincided with an alpha and beta band decrease. The final few hundred milliseconds of the epoch showed an alpha and beta band increase on frontal channels.

Figure 2 shows the power changes in the strongly constraining contexts. In these sentences, unexpected words elicited a stronger alpha and beta band power decrease than expected words. This effect had a posterior and left frontal distribution and was reflected in a cluster, $p=.016$. The cluster most consistently spanned frequencies from about 10 to $17 \mathrm{~Hz}$ between 500 and $700 \mathrm{~ms}$ across posterior, anterior, and left medial channels. ${ }^{1}$ In addition, unexpected words in strongly constraining sentences elicited a stronger theta increase than expected words. This effect was broadly distributed, with a fronto central maximum, and was reflected in a cluster, $p=.002$. The cluster included all channels, most consistently spanning frequencies from 3 to $8 \mathrm{~Hz}$ between about 300 and $700 \mathrm{~ms}$. (a)

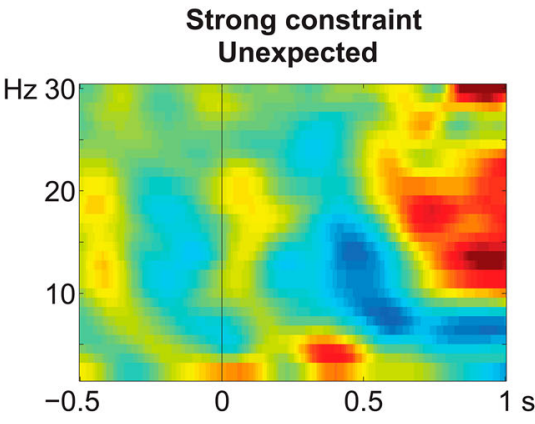

(b)

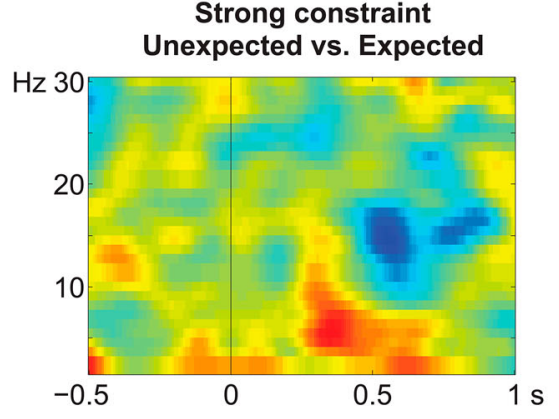

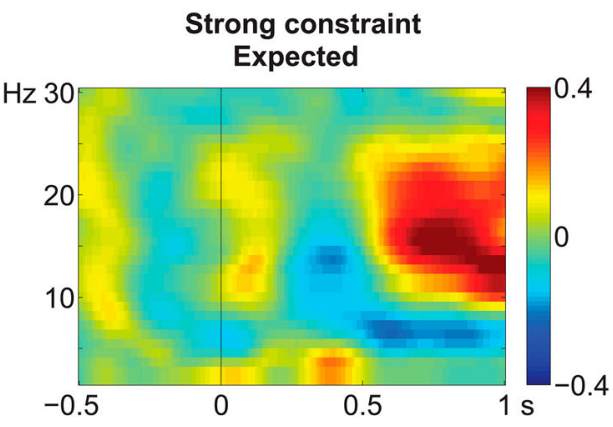

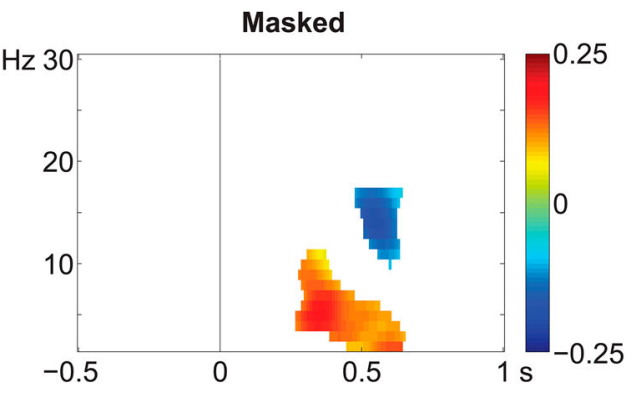

(c)
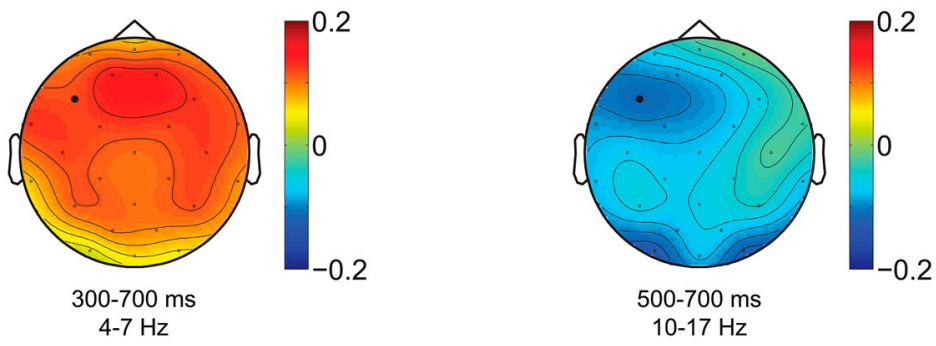

Figure 2. Grand average time-frequency plots of power changes in strongly constraining contexts at a frontal channel (LDFr, indicated with a black dot in the scalp maps). Time zero indicates the onset of the critical word. (a) Individual conditions. The colour scale indicates the proportion power change relative to a -500 to $-150 \mathrm{~ms}$ baseline. (b) Contrast between the conditions, showing a stronger theta band increase and alpha/beta band decrease in response to unexpected compared with expected words. Left: raw difference. Right: statistically thresholded difference. (c) Difference scalp topographies of the theta band increase and the alpha/beta band decrease. 
As Figure 3 shows, a similar alpha/beta power decrease occurred in weakly constraining sentences, more extended in time and somewhat different in frequency, but also with a posterior and left frontal distribution, cluster $p=.002$. The cluster spanned frequencies from 8 to $11 \mathrm{~Hz}$ on posterior and left medial channels between 300 and $500 \mathrm{~ms}$, and frequencies from around 17 to $22 \mathrm{~Hz}$ on medial and frontal channels between 400 and 800 ms. However, different from the pattern observed in the strongly constraining contexts, there was no theta band effect when comparing the unexpected and expected words in the weakly constraining contexts.

As shown in Figure 4, there was also an effect prior to the onset of the critical word. Relative to weakly constraining sentence contexts, strongly constraining sentence contexts showed a power decrease in the alpha band, sometimes including the beta band, which was reflected in a cluster, $p=.002$. The cluster included bursts of activity at varying frequencies over time from -1500 to $-500 \mathrm{~ms}$, most often encompassing frequencies between 7 and $12 \mathrm{~Hz}$ over frontal and central channels. Closer to word onset, there was also a burst between 16 and $24 \mathrm{~Hz}$ over left posterior and anterior channels. From around $-200 \mathrm{~ms}$ to word onset, the cluster consistently included a frequency band from 7 to $12 \mathrm{~Hz}$ over occipital and central channels, where the difference was visually most pronounced.

\section{Correlations}

We computed nonparametric Spearman correlations between several of the observed effects across participants. A number of the time-frequency effects exhibited a frontal maximum, reminiscent of the frontally distributed positivity in the ERPs. Therefore, one goal of the correlation analysis was to examine relationships between (a)

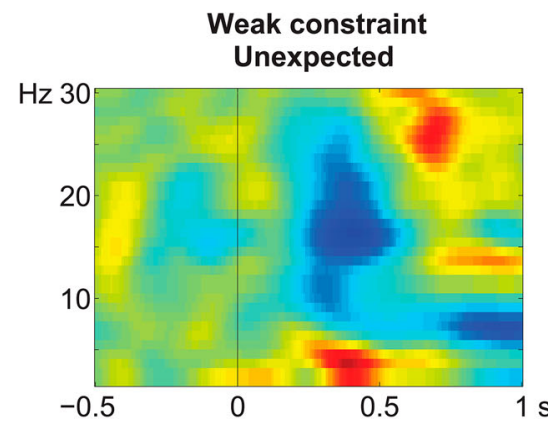

(b)

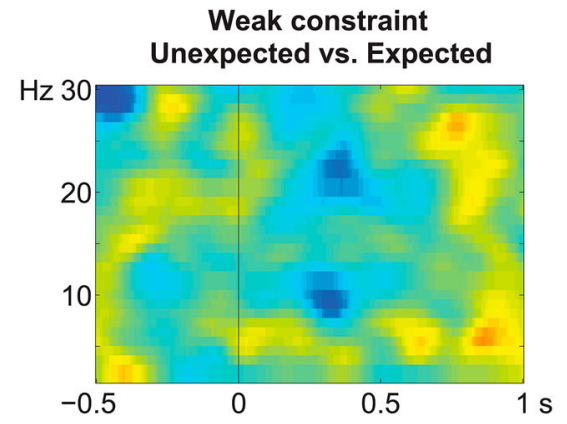

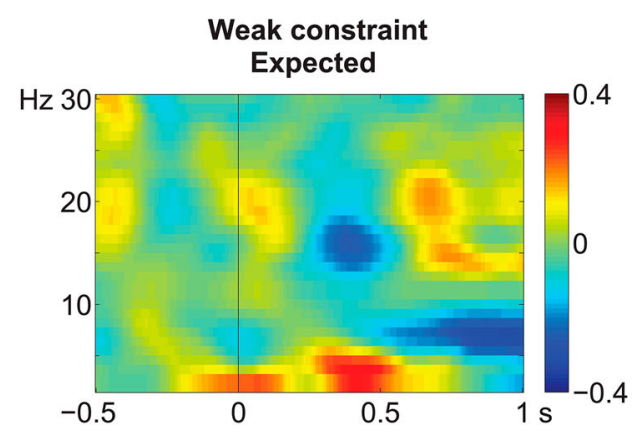

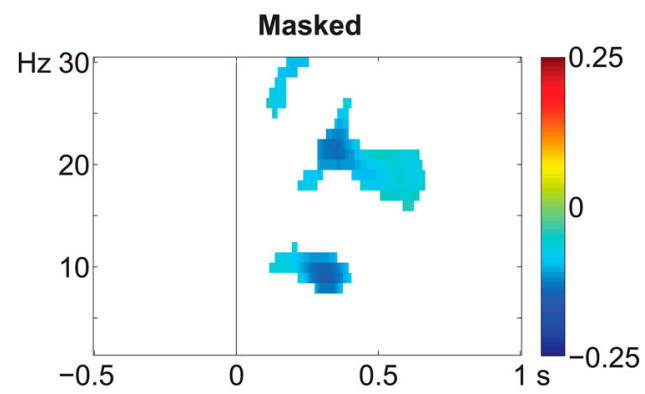

(c)
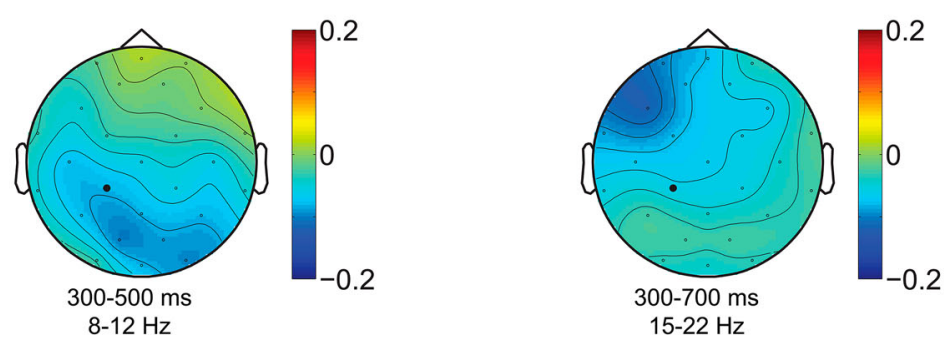

Figure 3. Grand average time-frequency plots of power changes in weakly constraining contexts at a left central channel (LMCe, indicated with a black dot in the scalp maps). Time zero indicates the onset of the critical word. (a) Individual conditions. The colour scale indicates the proportion power change relative to a -500 to -150 ms baseline. (b) Contrast between the conditions, showing a stronger alpha and beta band decrease in response to unexpected compared with expected words. Left: raw difference. Right: statistically thresholded difference. (c) Difference scalp topographies of the alpha band decrease (left) and the beta band decrease (right). 
(a)
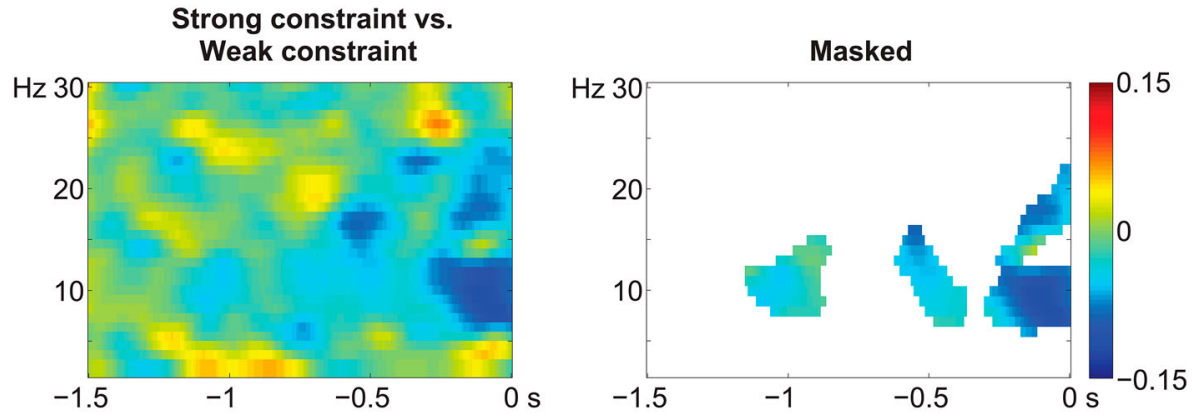

(b)

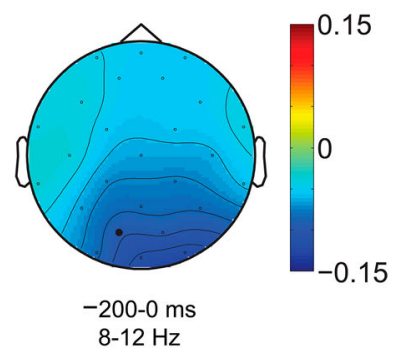

Figure 4. Grand average time-frequency plots of power changes in the strongly constraining versus the weakly constraining sentence contexts at a left occipital channel (LMOc, indicated with a black dot in the scalp map). Time zero indicates the onset of the critical word; negative values indicate time preceding its onset. (a) Contrast between the conditions, showing a stronger alpha and beta band decrease during strongly constraining compared with weakly constraining sentences. Left: raw difference. Right: statistically thresholded difference. (b) Difference scalp topography of the alpha band decrease.

the ERP data, in particular the late positive effect, and the various frontal effects in the time-frequency data. We focused on the difference between unexpected and expected words in strongly constraining contexts, because this contrast exhibited the theta effect. In Federmeier et al. (2007), the positivity was most clearly visible on three frontal channels (LMPf, RMPf, MiPf), and the frontal theta effect was present there too. Thus, to quantify the late positivity in individual subjects, the ERP amplitude difference between unexpected and expected words in strongly constraining sentences was averaged across these channels in a time window from 500 to $900 \mathrm{~ms}$ (following Federmeier et al., 2007; the pattern of results was the same for a $500-700 \mathrm{~ms}$ window, where the positivity overlaps most clearly with the theta effect). ${ }^{2}$ The frontal theta effect from the same contrast was extracted from the same channels and time window, averaged across the frequencies $4-7 \mathrm{~Hz}$. The alpha decrease elicited by unexpected relative to expected words showed a frontal as well as an occipital maximum (see Figure 2). These two aspects of the effect were examined separately, in both cases averaging across the frequencies $8-12 \mathrm{~Hz}$; the frontal alpha effect using the same three frontal channels from 500 to 900 $\mathrm{ms}$, and the occipital alpha effect using five occipital channels (LMOc, RMOc, LLOc, RLOc, and MiOc) from
400 to $800 \mathrm{~ms}$, based on the distribution of the effect and the detected clusters.

Another goal of the correlation analysis was to examine relationships between brain activity preceding the critical words and brain activity during the critical word. The pre-stimulus occipital alpha effect was measured as the difference in average power between the two levels of constraint (strongly constraining versus weakly constraining contexts) from 8 to $12 \mathrm{~Hz}$ in a time window from $-200 \mathrm{~ms}$ up to the critical word, averaged across the five occipital channels mentioned above. The resulting correlations are shown in Figure 5. All time-frequency effects were measured using power changes relative to the average across all conditions, as in the original analyses. The significant correlations were almost identical when the same analysis was done on difference scores of log-transformed raw power values (rho deviation 0.02 or less) or when the parametric Pearson's correlation coefficient was used (rho deviation 0.03 or less).

The correlations revealed three relationships. In the signal recorded during the processing of the critical words, participants with a greater ERP late positivity effect showed a greater frontal theta effect. Furthermore, a stronger constraint-dependent pre-stimulus occipital alpha decrease was associated with a weaker theta 


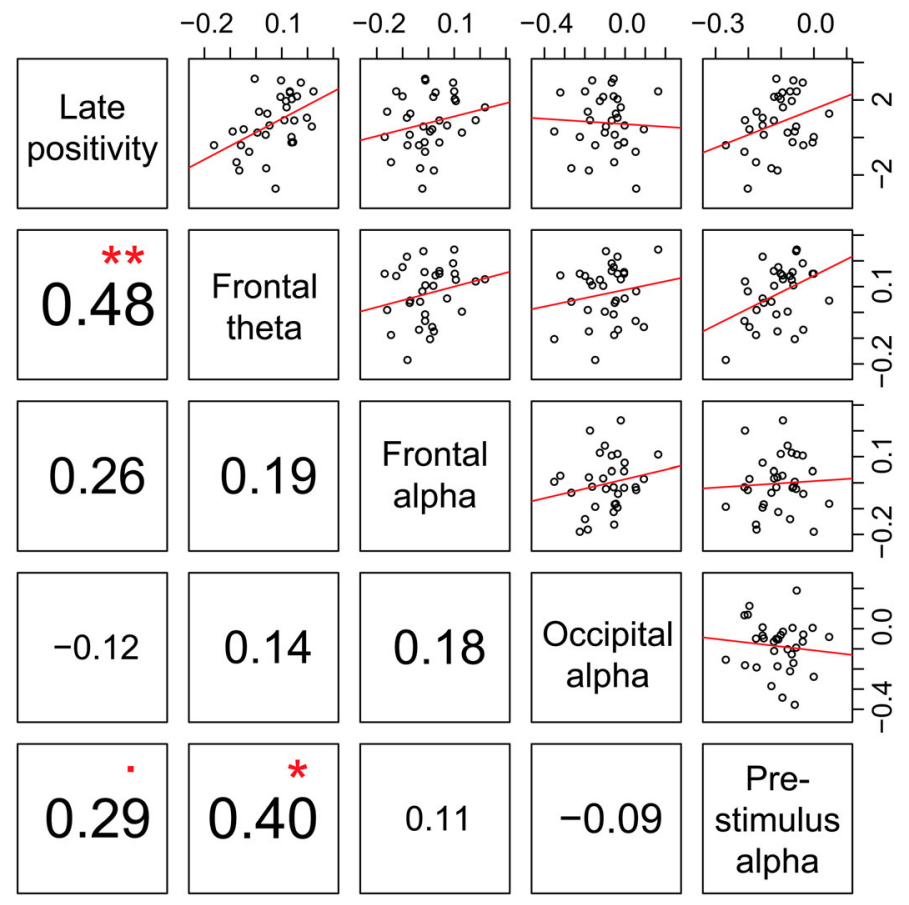

Figure 5. Correlations among individual participants' difference scores for strong constraint unexpected versus expected words (four top measures) and the pre-stimulus strong versus weak constraint effect (bottom measure). Lower left panels: Spearman correlation coefficients for each pair of measures, with the font size scaled to the absolute rho value. Upper right panels: scatterplots of each measure plotted against each other measure, along with linear regression lines. ${ }^{* *} p<.01,{ }^{*} p<.05, \cdot p<.1$.

increase to unexpected (versus expected) words. Stronger pre-stimulus occipital alpha was also, to some extent, associated with a smaller ERP late positivity difference between unexpected and expected words.

\section{Phase-locked and non-phase-locked power}

Because the correlations suggested a relationship between the late positive ERP effect and the frontal theta effect, we further examined to what extent the theta effect might be the spectral representation of the late positivity. This was done by distinguishing between phase-locked power (also known as "evoked" activity) and non-phase-locked power ("induced" activity; for discussion, see Cohen, 2014; Tallon-Baudry \& Bertrand, 1999). Non-phase-locked power was computed as follows: first, the condition-, channel-, and participant-specific unfiltered ERP was computed and subtracted from the corresponding individual trials in the time domain (Kalcher \& Pfurtscheller, 1995). Because the ERP primarily captures phase-locked activity (peaks and troughs that are aligned across trials), subtracting it should remove the phase-locked portion of the signal. Then, time-frequency analysis was performed as described in the Methods. Conversely, phase-locked power was computed by applying the same time-frequency analysis to the ERPs.
As shown in Figure 6 (right), there was a relatively short-lived theta band increase in the time-frequency representation of the ERPs, maximal at left central channels, which was detected as a cluster, $p=.002$. The cluster was broadly distributed over the scalp and, between 300 and $500 \mathrm{~ms}$, included frequencies from 3 to $6 \mathrm{~Hz}$ on most channels. From around 800 to $900 \mathrm{~ms}$, over left frontal and left parietal channels, it included the $2 \mathrm{~Hz}$ frequency; however, the reliability of power estimates from a 400 ms FFT window at such a low frequency can be considered questionable. The alpha/beta decrease observed in total power (Figure 2) was not present in phase-locked power. In contrast, in non-phase-locked power, both effects previously observed in the analysis of total power, namely the alpha and beta band power decrease and the theta band power increase, remained largely intact despite the subtraction of the ERPs. Both were detected as a cluster, $p=.01$ and $p=.002$, respectively. The cluster for the power increase most consistently encompassed frequencies between 3 and $6 \mathrm{~Hz}$ between 500 and $700 \mathrm{~ms}$, although earlier time points from 300 to $500 \mathrm{~ms}$ were included on some channels. An additional analysis (not shown), in which the ERPs were regressed out rather than subtracted from the individual trials, yielded essentially identical results.

Using the same data reduction settings as before, the late positivity still correlated across participants with the 
(a)



(b)

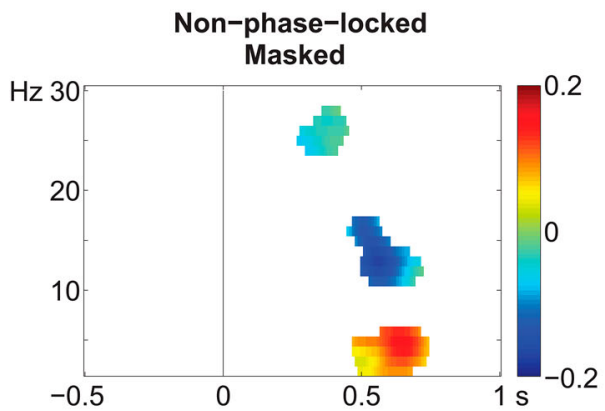

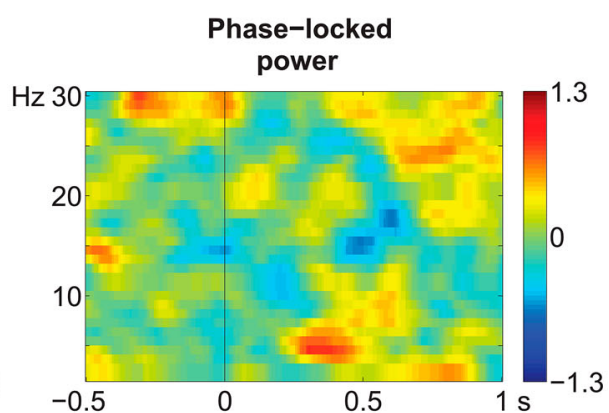

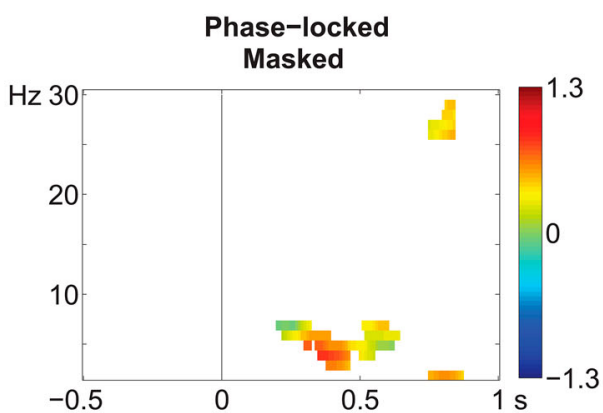

(c)
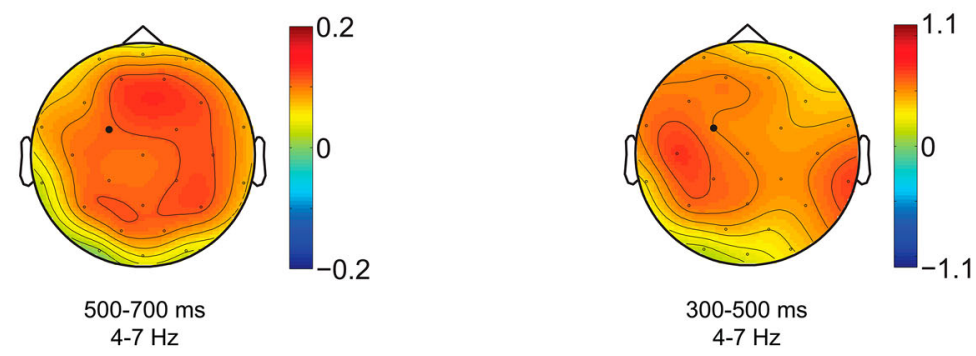

Figure 6. Grand average time-frequency plots of power changes in strongly constraining contexts, for the unexpected versus expected words, at a left frontal channel (LMFr, indicated with a black dot in the scalp maps). Time zero indicates the onset of the critical word. Left: non-phase-locked power (time-frequency analysis after subtracting the ERPs from the signal). Right: phase-locked power (timefrequency analysis of the ERP). (a) Raw difference, showing a stronger theta band increase and alpha/beta band decrease in response to unexpected compared with expected words. (b) Statistically thresholded difference. (c) Difference scalp topographies of the non-phaselocked frontal theta increase (left) and initial phase-locked theta increase (right).

frontal theta effect extracted from the non-phase-locked power values, $r=0.45, p=.01$, but interestingly, less so with the frontal theta effect in the phase-locked power values, $r=0.23, p=.201$. Taken together, the result pattern suggests that although the frontal theta effect and the late positive effect seem to be related signals, it appears unlikely that the frontal theta effect is fully explained by the signal captured in the ERPs.

\section{Discussion}

The present study aimed to examine brain mechanisms of predictive processing by characterising the spectral power dynamics in the EEG signal related to comprehending sentences that afforded differing degrees of predictability for sentence-final words. Thus, we examined effects elicited while participants read strongly and weakly constraining sentences for comprehension, and when they then encountered expected or unexpected but plausible words.

The results showed that, relative to expected words, unexpected words elicited an alpha and beta power decrease over posterior and anterior electrodes. Because this was similarly the case across levels of sentential constraint, this effect might not be an index of a prediction-related process per se. The results are consistent with earlier studies that observed similar effects in response to unexpected words in the form of anomalies (Luo et al., 2010; Wang, Jensen, et al., 2012). Thus, some aspects of processing unexpected words seem to be similar whether or not those words are encountered after the generation of strong predictions, and whether 
or not those words can ultimately be integrated with the context to produce a plausible message-level representation.

More strikingly, in strongly constraining contexts when readers would be more likely to form expectations - compared with expected words, unexpected words elicited a stronger, broadly distributed theta band power increase with a frontal maximum. This fits well with findings from nonverbal tasks, in which frontal theta activity has been linked to prediction error and cognitive control (e.g. Cavanagh \& Frank, 2014). Invoking control processes upon encountering input that disconfirms a likely prediction may be helpful from an adaptive learning perspective. The alternative interpretation of theta as reflecting lexical-semantic retrieval (e.g. Bastiaansen et al., 2005; Hald et al., 2006) is also a possibility: when a word was not expected, lexical-semantic retrieval processes may need to do more work compared with when a word could already be pre-activated. Importantly, the lexicalsemantic retrieval and cognitive control explanations are not mutually exclusive; in fact, theta activity has previously been characterised as reflecting controlled access to memory (Klimesch, Freunberger, \& Sauseng, 2010). One could speculate that additional control may be needed in order to retrieve the unexpected word from memory while preventing, discontinuing, or inhibiting retrieval of the expected word and/or revising an ongoing interpretation. To further determine the functional significance of theta activity in language processing, it would be interesting to see whether it is also related to error-based learning.

Participants with a greater ERP positivity difference between unexpected and expected words in strongly constraining contexts also showed a stronger frontal theta increase for the same contrast, opening up the possibility that the two effects could be different representations of the same underlying signal. Such a relationship would speak to the (in)dependence of the signals captured by each type of analysis (cf. Makeig et al., 2002; Mazaheri \& Jensen, 2006), as well as aid interpretation of the power changes by linking them to the more developed literature on ERPs. However, part of the frontal theta effect could be distinguished from a somewhat earlier and less frontally distributed theta increase in the ERPs. In addition, most of the theta effect remained visible after subtracting the ERPs from individual trials. Certain assumptions of this analysis can be debated, such as stationarity of the ERP waveform across trials, and these have been discussed in studies showing highly similar distinctions between phaselocked and non-phase-locked theta (Bastiaansen \& Hagoort, 2015; Cohen \& Donner, 2013). Fully disentangling the two signals would need manipulations that elicit each effect in the absence of the other, but based on the analyses presented here it appears unlikely that signals seen in the ERPs can fully account for the theta effect.

In addition to characterising effects at the critical word, as had also been done for the ERP signal in Federmeier et al. (2007), we were further able to characterise effects associated with the differential build-up of predictive information in strong and weak constraint sentences, prior to the onset of the final word. We found that, relative to weakly constraining sentences, strongly constraining sentences exhibited less alpha and beta power, especially over occipital electrode sites, prior to critical word onset. With respect to the issue of the domain specificity of prediction mechanisms, it is notable that pre-stimulus alpha effects have also been observed during the processing of nonverbal predictive sequences. In a study by Bidet-Caulet et al. (2012), participants saw sequences of three triangles with different orientations, which were predictive of a target triangle that they needed to detect. Prior to the occurrence of a decisive (third) triangle that signaled that a target would appear (relative to random sequences), alpha decreased over occipital channels, which was taken to reflect attentional preparation for the input. Several other studies suggest a role for alpha and beta power decreases in orienting attention to upcoming nonverbal stimuli (e.g. van Ede, de Lange, Jensen, \& Maris, 2011; Foxe, Simpson, \& Ahlfors, 1998; Thut, Nietzel, Brandt, \& Pascual-Leone, 2006; Worden, Foxe, Wang, \& Simpson, 2000). The participants in the present study may have relied on similar mechanisms while reading sentences.

The pre-stimulus alpha/beta decreases are also reminiscent of power decreases in the same frequency bands observed when people read constraining sentence frames while preparing to name an upcoming picture, which have been interpreted as reflecting facilitated access to memory and/or motor preparation (Piai et al., 2014; Piai et al., 2015). However, the EEG effect in Piai et al. (2014) seems to have a broader scalp distribution than the effect in the present study. Furthermore, its magnetic sources, especially in the beta band, included the left anterior temporal cortex (associated with access to concepts) only when participants prepared to name the picture, not when they prepared to judge the picture's predictability (Piai et al., 2015). At present, it is unclear whether the effect seen in the present study reflects different processes, or only different neuronal generator locations or strengths in implementing otherwise similar processes, such as some form of preparation.

Intuitively, one might have expected stronger alpha power decreases in weakly constraining than in strongly 
constraining contexts, because in weakly constraining contexts the upcoming word is going to provide more new information. However, the fact that in our study predictions were disconfirmed on half the trials (possibly increasing the need to prepare for the input) might play a role in inducing such preparation more saliently in the strongly constraining contexts instead. Indeed, exploring correlations among difference scores across subjects revealed that participants with a stronger constraint-driven pre-stimulus alpha decrease showed a weaker frontal theta increase in response to unexpected (versus expected) words in constraining contexts. This will need confirmation in future work: why would participants who use sentential constraint more show a weaker response to unexpected words in constraining contexts? The finding is suggestive of an interesting trade-off between predictability-related preparation and the consequences of having predictions then confirmed or disconfirmed. In particular, it suggests that increased preparation prior to the onset of the critical stimuli can mitigate the processing consequences of encountering unexpected input. Clearly, more research is needed, but on this account, the pre-stimulus occipital alpha increases might not reflect prediction per se, but rather preparation for the bottom-up input. In addition, this result alleviates possible concerns of visual differences between the sentence contexts driving the effects, an explanation that would not predict such a correlation (combined with the fact that in other studies that showed a similar pre-stimulus effect, visual aspects of the predictive stimuli were controlled).

Taken together, the findings appear consistent with the idea that making and assessing predictions during sentence comprehension relies on at least some mechanisms that are not specific to language. This adds to emerging evidence from anticipatory eye movements to visually presented objects while listening to predictive language. For instance, similar anticipatory eye movements have been observed during action observation and during listening to sentences describing those actions (Poljac, Dahlslätt, \& Bekkering, 2014). In addition, participants who show certain anticipatory eye movements based on predictive language context also seem to rely more on predictive arrow cues in a spatial attention task (Rommers, Meyer, \& Huettig, 2015).

In sum, the current study suggests that predictive language processing might recruit processes similar to those employed in nonverbal tasks, supporting proposals that language can take advantage of domain-general mechanisms. In particular, the results highlight how comprehenders can take advantage of predictive sentence contexts to prepare for upcoming input. Furthermore, when the input turns out to be unexpected, possibly yielding a prediction error signal, the brain appears to recruit general control processes to deal with the consequences of having that prediction disconfirmed. At a general level, this suggests that in further unravelling the cognitive and neurophysiological mechanisms underlying predictive language processing, it will be fruitful to continue to explore convergences across cognitive domains.

\section{Notes}

1. Descriptions of cluster extent are provided as an indication of the most likely contributors to each effect, but should not be taken to indicate effect onsets and offsets in time, space, or frequency. This is because the statistical test only controls the false alarm rate under a non-specific null hypothesis, namely that of no differences between the complete datasets in the two conditions (Maris, 2012).

2. It should be noted that, although the ERPs show a positive-going difference for this contrast (see Figure 2), in the original study (Federmeier et al., 2007) and its follow-ups, the focus had been on the contrast between unexpected words in strongly versus weakly constraining contexts, which more cleanly separates constraint and cloze probability. However, the expected versus unexpected comparison tested here is more similar to what has been examined in non-language tasks, and exploring similarities was a major aim of this study.

\section{Disclosure statement}

The authors report no potential conflict of interest.

\section{Funding information}

This work was supported by the $\mathrm{NIH}$ under Grant number AG026308 to K.D.F.; and by a James S. McDonnell Foundation Scholar Award to K.D.F.

\section{References}

Altmann, G. T. M., \& Kamide, Y. (1999). Incremental interpretation at verbs: Restricting the domain of subsequent reference. Cognition, 73(3), 247-264. doi:10.1016/S0010-0277 (99)00059-1

Altmann, G. T. M., \& Mirković, J. (2009). Incrementality and prediction in human sentence processing. Cognitive Science, 33 (4), 583-609. doi:10.1111/j.1551-6709.2009.01022.x

Arai, M., \& Keller, F. (2013). The use of verb-specific information for prediction in sentence processing. Language and Cognitive Processes, 28(4), 525-560. doi:10.1080/01690965. 2012.658072

Arnal, L. H., \& Giraud, A.-L. (2012). Cortical oscillations and sensory predictions. Trends in Cognitive Sciences, 16(7), 390398. doi:10.1016/j.tics.2012.05.003

Bastiaansen, M. C. M., \& Hagoort, P. (2015). Frequency-based segregation of syntactic and semantic unification during 
online sentence level language comprehension. Journal of Cognitive Neuroscience, 27(11), 2095-2107. doi:10.1162/ jocn_a_00829

Bastiaansen, M. C. M., Magyari, L., \& Hagoort, P. (2010). Syntactic unification operations are reflected in oscillatory dynamics during on-line sentence comprehension. Journal of Cognitive Neuroscience, 22, 1333-1347. doi:10.1162/jocn.2009.21283

Bastiaansen, M., van der Linden, M., ter Keurs, M., Dijkstra, T., \& Hagoort, P. (2005). Theta responses are involved in lexicalsemantic retrieval during language processing. Journal of Cognitive Neuroscience, 17(3), 530-541. doi:10.1162/ 0898929053279469

van Berkum, J. J. A., Brown, C. M., Zwitserlood, P., Kooijman, V., \& Hagoort, P. (2005). Anticipating upcoming words in discourse: Evidence from ERPs and reading times. Journal of Experimental Psychology: Learning, Memory, and Cognition, 31(3), 443-467. doi:10.1037/0278-7393.31.3.443

Bidet-Caulet, A., Barbe, P. G., Roux, S., Viswanath, H., Barthélémy, C., Bruneau, N., ... Bonnet-Brilhault, F. (2012). Dynamics of anticipatory mechanisms during predictive context processing. European Journal of Neuroscience, 36(7), 2996-3004. doi:10.1111/j.1460-9568.2012.08223.x

Carminati, M. N., Van Gompel, R. P. G., Scheepers, C., \& Arai, M. (2008). Syntactic priming in comprehension: The role of argument order and animacy. Journal of Experimental Psychology: Learning, Memory, and Cognition, 34, 10981110. doi:10.1037/a0012795

Cavanagh, J. F., \& Frank, M. J. (2014). Frontal theta as a mechanism for cognitive control. Trends in Cognitive Sciences, 18(8), 414-421. doi:10.1016/j.tics.2014.04.012

Cavanagh, J. F., Frank, M. J., Klein, T. J., \& Allen, J. J. B. (2010). Frontal theta links prediction errors to behavioral adaptation in reinforcement learning. Neurolmage, 49, 3198-3209. doi:10.1016/j.neuroimage.2009.11.080

Christiansen, M. H., \& Chater, N. (2015). The Now-or-never bottleneck: A fundamental constraint on language. Behavioral and Brain Sciences, 1-52. doi:10.1017/S0140525X1500031X

Clark, A. (2013). Whatever next? Predictive brains, situated agents, and the future of cognitive science. Behavioral and Brain Sciences, 36(3), 181-204. doi:10.1017/s0140525x12000477

Cohen, M. X. (2014). Analyzing neural time series data: theory and practice. MIT Press.

Cohen, M. X., \& Donner, T. H. (2013). Midfrontal conflict-related theta-band power reflects neural oscillations that predict behavior. Journal of Neurophysiology, 110(12), 2752-2763. doi:10.1152/jn.00479.2013

Davidson, D. J., \& Indefrey, P. (2007). An inverse relation between event-related and time-frequency violation responses in sentence processing. Brain Research, 1158, 8192. doi:10.1016/j.brainres.2007.04.082

Dell, G. S., \& Chang, F. (2014). The P-chain: Relating sentence production and its disorders to comprehension and acquisition. Philosophical Transactions of the Royal Society B: Biological Sciences, 369(1634), 20120394. doi:10.1098/rstb.2012.0394

DeLong, K. A., Quante, L., \& Kutas, M. (2014). Predictability, plausibility and two late ERP positivities during written sentence comprehension. Neuropsychologia, 61, 150-162. doi:10.1016/j.neuropsychologia.2014.06.016

DeLong, K. A., Urbach, T. P., \& Kutas, M. (2005). Probabilistic word pre-activation during language comprehension inferred from electrical brain activity. Nature Neuroscience, 8 (8), 1117-1121. doi:10.1038/nn1504 van Ede, F., de Lange, F. P., Jensen, O., \& Maris, E. (2011). Orienting attention to an upcoming tactile event involves a spatially and temporally specific modulation of sensorimotor alpha- and beta-band oscillations. Journal of Neuroscience, 31, 2016-2024. doi:10.1523/JNEUROSCI.5630-10.2011

Federmeier, K. D. (2007). Thinking ahead: The role and roots of prediction in language comprehension. Psychophysiology, 44, 491-505. doi:10.1111/j.1469-8986.2007.00531.x

Federmeier, K. D., \& Kutas, M. (1999). A rose by any other name: Long-term memory structure and sentence processing. Journal of Memory and Language, 41(4), 469-495. doi:10. 1006/jmla.1999.2660

Federmeier, K. D., Wlotko, E., De Ochoa-Dewald, E., \& Kutas, M. (2007). Multiple effects of sentential constraint on word processing. Brain Research, 1146, 75-84. doi:10.1016/j.brainres. 2006.06.101

Foxe, J. J., Simpson, G. V., \& Ahlfors, S. P. (1998). Parieto-occipital $\sim 10 \mathrm{~Hz}$ activity reflects anticipatory state of visual attention mechanisms. Neuroreport, 9(17), 3929-3933. doi:10.1097/ 00001756-199812010-00030

Friederici, A. D., \& Singer, W. (2015). Grounding language processing on basic neurophysiological principles. Trends in Cognitive Sciences, 19(6), 329-338. doi:10.1016/j.tics.2015.03.012

Fries, P. (2005). A mechanism for cognitive dynamics: Neuronal communication through neuronal coherence. Trends in Cognitive Sciences, 9(10), 474-480. doi:10.1016/j.tics.2005. 08.011

Friston, K. (2010). The free-energy principle: A unified brain theory? Nature Reviews Neuroscience, 11(2), 127-138. doi:10. 1038/nrn2787

Hald, L. A., Bastiaansen, M. C. M., \& Hagoort, P. (2006). EEG theta and gamma responses to semantic violations in online sentence processing. Brain and Language, 96(1), 90-105. doi:10.1016/j.bandl.2005.06.007

Kalcher, J., \& Pfurtscheller, G. (1995). Discrimination between phase-locked and non-phase-locked event-related EEG activity. Electroencephalography and Clinical Neurophysiology, 94, 381-384. doi:10.1016/0013-4694(95)00040-6

Kamide, Y. (2008). Anticipatory processes in sentence processing. Language and Linguistics Compass, 2(4), 647-670. doi:10.1111/j.1749-818X.2008.00072.x

Kleinschmidt, D. F., \& Jaeger, T. F. (2015). Robust speech perception: Recognize the familiar, generalize to the similar, and adapt to the novel. Psychological Review, 122(2), 148. doi:10.1037/a0038695

Klimesch, W., Freunberger, R., \& Sauseng, P. (2010). Oscillatory mechanisms of process binding in memory. Neuroscience \& Biobehavioral Reviews, 34(7), 1002-1014. doi:10.1016/j. neubiorev.2009.10.004

Kutas, M., DeLong, K. A., \& Smith, N. J. (2011). A look around at what lies ahead: Prediction and predictability in language processing. Predictions in the Brain: Using Our Past to Generate a Future, 190-207. doi:10.1093/acprof:oso/ 9780195395518.003.0065

Kutas, M., \& Hillyard, S. A. (1980). Reading senseless sentences: Brain potentials reflect semantic incongruity. Science, 207, 203-205. doi:10.1126/science.7350657

Laszlo, S., \& Federmeier, K. D. (2009). A beautiful day in the neighborhood: An event-related potential study of lexical relationships and prediction in context. Journal of Memory and Language, 61, 326-338. doi:10.1016/j.jml. 2009.06.004 
Levy, R. (2008). Expectation-based syntactic comprehension. Cognition, 106(3), 1126-1177. doi:10.1016/j.cognition.2007.05.006

Lewis, A. G., Wang, L., \& Bastiaansen, M. C. M. (2015). Fast oscillatory dynamics during language comprehension: Unification versus maintenance and prediction? Brain \& Language, 148, 51-63. doi:10.1016/j.bandl.2015.01.003

Luck, S. J., \& Kappenman, E. S. (Eds.). (2011). The Oxford handbook of event-related potential components. New York: Oxford University Press. doi:10.1093/oxfordhb/ 9780195374148.001.0001

Luo, Y., Zhang, Y., Feng, X., \& Zhou, X. (2010). Electroencephalogram oscillations differentiate semantic and prosodic processes during sentence reading. Neuroscience, 169, 654-664. doi:10. 1016/j.neuroscience.2010.05.032

Luu, P., \& Tucker, D. M. (2001). Regulating action: Alternating activation of midline frontal and motor cortical networks. Clinical Neurophysiology, 112, 1295-1306. doi:10.1016/ S1388-2457(01)00559-4

Makeig, S., Westerfield, M., Jung, T. P., Enghoff, S., Townsend, J., Courchesne, E., \& Sejnowski, T. J. (2002). Dynamic brain sources of visual evoked responses. Science, 295(5555), 690-694. doi:10.1126/science.1066168

Maris, E. (2012). Statistical testing in electrophysiological studies. Psychophysiology, 49(4), 549-565. doi:10.1111/j. 1469-8986.2011.01320.x

Maris, E., \& Oostenveld, R. (2007). Nonparametric statistical testing of EEG- and MEG-data. Journal of Neuroscience Methods, 164(1), 177-190. doi:10.1016/j.jneumeth.2007.03.024

Mazaheri, A., \& Jensen, O. (2006). Posterior a activity is not phase-reset by visual stimuli. Proceedings of the National Academy of Sciences of the United States of America, 103(8), 2948-2952. doi:10.1073/pnas.0505785103

Oostenveld, R., Fries, P., Maris, E., \& Schoffelen, J.-M. (2011). Fieldtrip: Open source software for advanced analysis of MEG, EEG, and invasive electrophysiological data. Computational Intelligence and Neuroscience, 2011, 9. doi:10.1155/2011/156869

Peña, M., \& Melloni, L. (2012). Brain oscillations during spoken sentence processing. Journal of Cognitive Neuroscience, 24, 1149-1164. doi:10.1162/jocn_a_00144

van Petten, C., \& Luka, B. J. (2012). Prediction during language comprehension: Benefits, costs, and ERP components. International Journal of Psychophysiology, 83, 176-190. doi:10.1016/j.ijpsycho.2011.09.015

Piai, V., Roelofs, A., \& Maris, E. (2014). Oscillatory brain responses in spoken word production reflect lexical frequency and sentential constraint. Neuropsychologia, 53, 146-156. doi:10. 1016/j.neuropsychologia.2013.11.014

Piai, V., Roelofs, A., Rommers, J., \& Maris, E. (2015). Beta oscillations reflect memory and motor aspects of spoken word production. Human Brain Mapping, 36(7), 2767-2780. doi:10.1002/hbm.22806

Pickering, M. J., \& Garrod, S. (2013). An integrated theory of language production and comprehension. Behavioral and Brain Sciences, 36(04), 329-347. doi:10.1017/ S0140525X12001495

Poljac, E., Dahlslätt, K., \& Bekkering, H. (2014). Shared predictive decision-making mechanisms in action and language. Language, Cognition and Neuroscience, 29(4), 424-434. doi:10.1080/01690965.2013.791702

Rayner, K. (1998). Eye movements in reading and information processing: 20 years of research. Psychological Bulletin, 124 (3), 372-422. doi:10.1037/0033-2909.124.3.372
Robb, M. P., Maclagan, M. A., \& Chen, Y. (2004). Speaking rates of American and New Zealand varieties of English. Clinical Linguistics \& Phonetics, 18(1), 1-15. doi:10.1080/ 0269920031000105336

Rommers, J., Dijkstra, T., \& Bastiaansen, M. C. M. (2013). Contextdependent semantic processing in the human brain: Evidence from idiom comprehension. Journal of Cognitive Neuroscience, 25(5), 762-776. doi:10.1162/jocn_a_00337

Rommers, J., Meyer, A. S., \& Huettig, F. (2015). Verbal and nonverbal predictors of language-mediated anticipatory eye movements. Attention, Perception, \& Psychophysics, 77(3), 720-730. doi:10.3758/s13414-015-0873-x

Rommers, J., Meyer, A. S., Praamstra, P., \& Huettig, F. (2013). The contents of predictions in sentence comprehension: Activation of the shape of objects before they are referred to. Neuropsychologia, 51(3), 437-447. doi:10.1016/j. neuropsychologia.2012.12.002

Singer, W. (1993). Synchronization of cortical activity and its putative role in information processing and learning. Annual Review of Physiology, 55, 349-374. doi:10.1146/ annurev.physiol.55.1.349

Staub, A., \& Clifton Jr, C., (2006). Syntactic prediction in language comprehension: Evidence from either ... or. Journal of Experimental Psychology: Learning, Memory, and Cognition, 32, 425-436. doi:10.1037/0278-7393.32.2.425

Tallon-Baudry, C., \& Bertrand, O. (1999). Oscillatory gamma activity in humans and its role in object representation. Trends in Cognitive Sciences, 3, 151-162. doi:10.1016/S13646613(99)01299-1

Thut, G., Nietzel, A., Brandt, S. A., \& Pascual-Leone, A. (2006). aBand electroencephalographic activity over occipital cortex indexes visuospatial attention bias and predicts visual target detection. The Journal of Neuroscience, 26(37), 94949502. doi:10.1523/JNEUROSCI.0875-06.2006

van de Vijver, I., Ridderinkhof, K. R., \& Cohen, M. X. (2011). Frontal oscillatory dynamics predict feedback learning and action adjustment. Journal of Cognitive Neuroscience, 23 (12), 4106-4121. doi:10.1162/jocn_a_00110

Wang, L., Jensen, O., Van den Brink, D., Weder, N., Schoffelen, J.M., Magyari, L., ... Bastiaansen, M. C. M. (2012). Beta oscillations relate to the $\mathrm{N} 400 \mathrm{~m}$ during language comprehension. Human Brain Mapping, 33, 2898-2912. doi:10.1002/ hbm. 21410

Wang, L., Zhu, Z., \& Bastiaansen, M. C. M. (2012). Integration or predictability? A further specification of the functional role of gamma oscillations in language comprehension. Frontiers in Psychology, 3, 187. doi:10.3389/fpsyg.2012.00187

Wicha, N. Y. Y., Moreno, E. M., \& Kutas, M. (2004). Anticipating words and their gender: An event-related brain potential study of semantic integration, gender expectancy, and gender agreement in Spanish sentence reading. Journal of Cognitive Neuroscience, 16(7), 1272-1288. doi:10.1162/ 0898929041920487

Willems, R. M., Frank, S. L., Nijhof, A. D., Hagoort, P., \& Van den Bosch, A. (2015). Prediction during natural language comprehension. Cerebral Cortex, bhv075. doi:10.1093/ cercor/bhv075

Worden, M. S., Foxe, J. J., Wang, N., \& Simpson, G. V. (2000). Anticipatory biasing of visuospatial attention indexed by retinotopically specific a-band electroencephalography increases over occipital cortex. Journal of Neuroscience, 20 (RC63), 1-6. 